\title{
PROPIEDAD INDUSTRIAL Y RESULTADOS EMPRESARIALES EN LAS AUTORIDADES PORTUARIAS ESPAÑOLAS DEL SIGLO XXI
}

\author{
REMEDIOS RAMÓN-DANGLA \\ Departamento Economía Financiera y Contabilidad, UNIVERSIDAD DE ALICANTE, ESPAÑA \\ e-mail: remedios.ramon@ua.es \\ LYDIA BARES-LÓPEZ \\ Departamento Economía General, UNIVERSIDAD DE CÁDIZ, ESPAÑA \\ e-mail: lydia.bares@uca.es \\ CRISTINA BAÑÓN-CALATRAVA \\ Departamento Economía Financiera y Contabilidad, UNIVERSIDAD DE ALICANTE, ESPAÑA \\ e-mail: c.banon@ua.es
}

\section{RESUMEN}

El objetivo de este artículo es analizar el impacto de la innovación sobre los resultados económicos de las Autoridades Portuarias españolas en el periodo 2008-2015. El Sistema Portuario español está compuesto por 46 puertos de interés general organizados en 28 Autoridades Portuarias, que se han clasificado en grandes, medianas y pequeñas dependiendo de la cifra neta de negocios. Como variable proxy de la innovación se ha utilizado la cuenta de propiedad industrial, obtenida de las cuentas anuales publicadas en los Boletines Oficiales del Estado. Los resultados muestran que existe una correlación significativa entre la inversión en propiedad industrial, y, por tanto, entre el esfuerzo en innovación y la cifra neta de negocios de las Autoridades Portuarias.

Palabras clave: Autoridades Portuarias, Innovación, Propiedad Industrial, Resultados Empresariales.

\section{Industrial property and business results in the Spanish port authorities of the 21st century}

\begin{abstract}
The objective of this article is to analyse the impact of innovation on the economic results of the Spanish Port Authorities in the period 20082015. The Spanish Port System is composed of 46 ports of general interest organized in 28 Port Authorities, which have been classified into large, medium and small depending on the net business amount. The Industrial Property account, obtained from the Annual Accounts and published in the Official State Gazettes has been used as a proxy variable for innovation. The results show that there is a significant correlation between the investment in Industrial Property, and, therefore, between the effort in innovation and the net business amount of the Port Authorities.
\end{abstract}

Keywords: Port Authorities, Innovation, Industrial Property, Business Results.

Clasificación JEL: L92; M41; 033. 


\section{INTRODUCCIÓN}

El proceso de globalización de la economía y del comercio internacional ha conllevado la necesidad de innovar y modernizar las redes de transporte, particularmente, el marítimo. En este contexto, y con el objetivo de atraer tráficos marítimos internacionales y mejorar su posicionamiento económico, los puertos han necesitado mejorar su eficacia y competitividad, considerándose la innovación como la principal fuente de ventaja competitiva.

La literatura es prolija intentando establecer relaciones entre innovación y resultado empresarial (Crossan y Apaydin, 2010), sin embargo, la dificultad y la diversidad de conclusiones radica en la definición y medición de la innovación (Somohano et al., 2018). Una innovación es "un producto o proceso nuevo o mejorado (o una combinación de los mismos) que difiere significativamente de los productos o procesos anteriores de la organización y que se ha puesto a disposición de los usuarios potenciales (producto) o se ha puesto en uso por la organización (proceso)" (OECD-Oslo Manual, 2018:60). Por otra parte, también puede consistir en "sacar al mercado productos nuevos o muy mejorados o con encontrar formas más efectivas (mediante la puesta en práctica de procesos y métodos nuevos o muy mejorados) de comercializar los productos" (OECD-Manual de Frascati, 2015:64). De ahí que, predominantemente en la investigación se utilice, siguiendo a Mahnazmoradi (2017) los gastos en $\mathrm{I}+\mathrm{D}$ y la inversión en propiedad industrial ${ }^{\bullet}$ como una aproximación a las innovaciones de servicio, de procesos o administrativas. Sin embargo, no es el único autor que utiliza la variable de propiedad industrial, puesto que en la literatura científica las patentes ${ }^{\bullet}$ se consideran como una de las variables proxy de la innovación, sobre todo con el objetivo de analizar la relación entre innovación y rendimientos económicos de las empresas (Griliches, 1990; Harhoff et al., 2003; Lanjouw y Schankerman, 2004).

Por otra parte, también existen diversos trabajos que estudian el efecto sinérgico de los puertos españoles o sus Autoridades Portuarias (AAPP) sobre la economía local como pueden ser los trabajos de Acosta et al., (2011) o Castillo Manzano (2001), que evalúan el impacto económico de los puertos gaditanos en su entorno, o bien, para el caso de Santander el trabajo de Coto Millán et al., (2010), incluso el de Artal-Tur et al., (2015) que analiza mediante tablas Input-Output el impacto económico del puerto de Cartagena en la región de Murcia.

Sin embargo, hay menos estudios que pretendan analizar la innovación tecnológica de las AAPP a través de la cuenta (203) "propiedad industrial" y sus efectos sobre sus ingresos o cifra neta de negocios. Nuestro objetivo es, por tanto, contribuir con esta área de conocimiento y analizar la propiedad industrial de las AAPP españolas en los últimos años e intentar mostrar si existen vínculos o no, con sus resultados económicos.

El trabajo se estructura de la siguiente forma. En el segundo epígrafe explicaremos el funcionamiento de las AAPP españolas y analizaremos sus posibles actividades de innovación teniendo en cuenta la revisión bibliográfica, lo que nos dará pie a formular algunas hipótesis. En un tercer epígrafe describiremos la metodología y fuentes utilizadas. Posteriormente analizaremos los resultados y estableceremos unas conclusiones, así como las futuras líneas de investigación.

\footnotetext{
- Según Griliches (1998), los gastos de I+D se utilizan como inputs del proceso de innovación en la empresa, siendo el output el resultado del proceso de innovación, esto es, la propiedad industrial. En contabilidad, el stock de los importes invertidos en propiedad industrial se encuentra recogido en la cuenta 203 del cuadro de cuentas: Propiedad Industrial y forma parte del activo no corriente dentro del Balance.

- La propiedad industrial está formada por los diseños industriales, marcas y nombres comerciales (signos distintivos), patentes y modelos de utilidad, y topografias de semiconductores, por lo tanto, las patentes son una parte de la propiedad industrial.
} 


\section{AUTORIDADES PORTUARIAS E INNOVACIÓN. UNA REVISIÓN BIBLIOGRÁFICA Y ELABORACIÓN DE HIPÓTESIS}

\subsection{Autoridades Portuarias y su tratamiento contable}

Un puerto marítimo es definido como "el conjunto de espacios terrestres, aguas marítimas e instalaciones que, situado en la ribera de la mar o de las rías, reúne las condiciones físicas, naturales o artificiales y de organización que le permiten la realización de operaciones de tráfico portuario, y es autorizado para el desarrollo de estas actividades por la Administración competente" (Artículo 2 del Real Decreto Legislativo 2/2011, de 5 de septiembre, por el que se aprueba el Texto Refundido de la Ley de Puertos del Estado y de la Marina Mercante). Por ello, como indican entre muchos, Dooms et al., (2010) o Acosta et al., (2007) los puertos se configuran como grandes centros de actividad económica y logística, que favorecen el desarrollo económico local y regional a través de sus efectos multiplicadores.

Por los puertos entran y salen la mayor parte de mercancías destinadas a la importación y/o exportación, de ahí que, en países como España, con casi 8.000 kilómetros de línea de costa, se muevan miles de toneladas al año (Tabla 1), lo que refleja el importante papel que juegan para la economía nacional.

Tabla 1

Total del Tráfico Portuario en España. Miles de Toneladas.

\begin{tabular}{|l|l|l|l|l|l|l|l|}
\hline $\mathbf{2 0 0 8}$ & $\mathbf{2 0 0 9}$ & $\mathbf{2 0 1 0}$ & $\mathbf{2 0 1 1}$ & $\mathbf{2 0 1 2}$ & $\mathbf{2 0 1 3}$ & $\mathbf{2 0 1 4}$ & $\mathbf{2 0 1 5}$ \\
\hline 473,82 & 413,04 & 432,55 & 457,96 & 475,20 & 458,87 & 482,08 & 502,43 \\
\hline
\end{tabular}

Fuente: Sistema Portuario de Titularidad Estatal. Anuarios Estadísticos 2008-2015. (Ministerio de Fomento, 2018)

Ser el país de la Unión Europea con más línea de costa hace que nuestro Sistema Portuario esté formado por 46 puertos, gestionados por 28 AAPP y que, en su conjunto, gestionan más del $60 \%$ de las exportaciones y del $85 \%$ de las importaciones, lo que representa más de la mitad del comercio con la Unión Europea y prácticamente la totalidad del comercio con terceros países (Ministerio de Fomento, 2018). Además, la actividad portuaria aporta más de un $1 \%$ al PIB nacional y genera más de 35.000 empleos directos (Ministerio de Fomento, 2018).

El crecimiento del comercio exterior, como consecuencia de la recuperación económica, debería provocar un crecimiento de las actividades portuarias donde la capacidad de adaptación a los nuevos requerimientos de la demanda y la innovación pueden ser las claves para mejorar el rendimiento de los puertos.

El hecho de disponer de unas buenas infraestructuras, unido a unas prestaciones de servicios fiables y de calidad y a una innovación tecnológica, podrían estar en la base de una mejora de la competitividad de los puertos españoles, lo que supondría un mayor crecimiento local por efectos sinérgicos. En este sentido, la política de gestión portuaria es ejecutada por el Organismo Público Puertos del Estado que depende del Ministerio de Fomento y se gestiona a través del Real Decreto Legislativo 2/2011, de 5 de septiembre, y sus sucesivas modificaciones.

Desde el punto de vista económico y de rendición de cuentas, las AAPP se rigen, como cualquier administración pública, por el Derecho Administrativo y por la Ley de Patrimonio de las Administraciones Públicas (Ley 33/2003, de 3 de noviembre, del Patrimonio de las Administraciones Públicas) que las clasifica jurídicamente como "Entidades Estatales de Derecho Público" y, en su art 166.1.b) establece su régimen económico-financiero y presupuestario al determinar que "las Entidades de Derecho Público vinculadas a la Administración General del Estado o a sus organismos públicos cuyos ingresos provengan, al menos en un 50 por 100 de operaciones realizadas en el mercado serán regidas por Plan General de Contabilidad de la empresa española".

Por tanto, aunque la mayor parte de los bienes del activo no corriente de las AAPP son de dominio público, están habilitadas para disponer de amplios poderes de administración sobre ellos y percibir como propios los ingresos que se deriven de su gestión. De ahí, que el art. 39 del Real Decreto Legislativo 2/2011, de 5 de septiembre, por el que se aprueba el Texto Refundido de la Ley de Puertos del Estado y de la Marina Mercante establezca que "la contabilidad de las AAPP se ajuste a las disposiciones del Código de Comercio, a las del Plan General de Contabilidad de 2007 y a las demás 
que sean de aplicación. Para garantizar la homogeneidad contable, Puertos del Estado establece las directrices relativas a los criterios valoración, normas de elaboración y estructura de las cuentas anuales, que deben ser aplicadas por las AAPP". El ejercicio económico es por periodos anuales desde el 1 de enero hasta el 31 de diciembre de cada año".

Además, el mismo art 39 y el art. 40 del Real Decreto Legislativo 2/2011, de 5 de septiembre, por el que se aprueba el Texto Refundido de la Ley de Puertos del Estado y de la Marina Mercante determina que las Cuentas Anuales, que se componen de Balance, Pérdidas y Ganancias, Estado de Cambios en el Patrimonio Neto, Estado de Flujos de Efectivo y Memoria, "se deben formular en el plazo máximo de tres meses desde el cierre del ejercicio económico, se rendirán de acuerdo con los principios y normas de contabilidad recogidos en el Plan General de Contabilidad vigente para la empresa española y deberán ser aprobadas antes de finalizar el primer semestre del siguiente año por el Consejo de Administración de la AAPP o por el Consejo Rector de Puertos del Estado, en el caso de las cuentas anuales consolidadas del sistema portuario". Una vez aprobadas las Cuentas Anuales por la Junta del Puerto o Consejo de Administración, serán auditadas por la Intervención General de la Administración del Estado (IGAE) con el objetivo de que se mantenga la estabilidad presupuestaria estatal.

La formulación y presentación de cuentas de las AAPP conforme al Plan General de Contabilidad de empresa de 2007 nos permite analizar dicha información contable y extraer conclusiones como si se tratara de una empresa privada. Por lo que siguiendo a Amat (2000), Gonzalo et al., (2000), Sánchez (2002) Godoy (2004), Garrido e Íñiguez (2012) haremos especial hincapié en la evaluación de la solvencia, de la situación financiera y de los resultados empresariales como variables fundamentales para la toma de decisiones de los agentes económicos.

\subsection{La innovación industrial y sus efectos}

A lo largo de la historia, han existido diversas teorías que han tratado de explicar la relación entre producción tecnológica y desarrollo económico. El primer economista que propuso un modelo económico para explicar dicho vínculo fue Solow y se denominó teoría neoclásica o de crecimiento exógeno. Solow (1957) realizó un análisis causal entre los factores productivos y el crecimiento en la economía de Estados Unidos en el periodo 1909-1949, utilizando la función Cobb-Douglas. Su principal conclusión fue que solamente el $12,5 \%$ del crecimiento del producto interior bruto estaba explicado por los incrementos en el capital y trabajo, por lo que el $87,5 \%$ era debido a una variable exógena denominada "progreso técnico", y, por lo tanto, principal fuente de crecimiento económico a largo plazo.

Sin embargo, como esta teoría no explicaba los determinantes económicos de esa variable, se estableció la teoría del crecimiento endógeno o nueva teoría del crecimiento. Romer (1986) y Lucas (1988) fueron los autores que fijaron las bases de este modelo, el cual se fundamentaba en la idea de que esta variable era endógena, es decir, se enmarcaba dentro del proceso de producción, en concreto, dentro de la innovación desarrollada por las empresas, dando origen a la creación de nuevo conocimiento.

Posteriormente, se desarrollaron las teorías evolucionistas o neo-shumpeterianas, las cuales consideraban la innovación como un proceso en constante evolución, siendo una variable endógena a la empresa, cuyas principales características hacían referencia a su carácter acumulativo, a su diversidad e incertidumbre, así como a la apropiabilidad y a la distinción entre conocimiento codificado y tácito, en las que se integran factores de índole organizacional, institucional y de aprendizaje (Freeman, 1994). Así mismo, sus principales contribuciones empíricas se refieren a las políticas gubernamentales que tienen como propósito la generación y transferencia de la innovación, desencadenado efectos positivos en la economía, a nivel macro y microeconómico.

Según Sánchez y Salazar (2010), la innovación origina beneficios macroeconómicos. Para su explicación, parten de la función de demanda agregada de Keynes (1936), que se define como la suma de todos los gastos en bienes y servicios llevados a cabo por las familias, empresas, sector público y sector exterior. 
Ahora bien, la innovación no sólo es positiva para el desarrollo económico de las naciones, sino que, además, como concluye Metcalfe (1995), desde el lado microeconómico también provoca importantes beneficios tanto en instituciones públicas como en organizaciones privadas.

Por el lado de las instituciones públicas y siguiendo a Franzoni y Scellato (2011), cuando un organismo público invierte en innovación y en actividades de investigación y desarrollo (I+D) se beneficia de una serie de efectos. Por una parte, se produce una mejora en el proceso de transferencia de tecnología al permitir que dichas entidades licencien su propiedad intelectual y, como consecuencia, se genera un incremento en sus recursos financieros provenientes de las regalías de los contratos de licencias. Por otra parte, existe la posibilidad de crear empresas de base tecnológica (EBT), que, además, podrían suponer una confluencia entre las investigaciones realizadas por entidades públicas y empresas, lo que ampliaría el efecto multiplicador de la inversión en innovación, $\mathrm{y}$, por último, puede obtener una mejora en la responsabilidad de la investigación realizada y de su valoración.

Por el lado de las empresas, Grandón y Rodríguez (1992) fueron pioneros en España al vincular la innovación tecnológica con el crecimiento empresarial, medido a través del volumen de ventas. Su metodología se basaba en datos de panel y una muestra de 53 empresas en el periodo 1973-1981. Concluyeron que el gasto en investigación y desarrollo estaba relacionado directa y significativamente con el volumen de ventas en la sub-etapa 1979-1981. Por el contrario, no encontraron ninguna relación entre las variables durante el periodo 1975-1978. Esa diferencia entre las dos sub-etapas la relacionaron con la crisis del petróleo de 1979. Lo que nos permite formular nuestra primera hipótesis:

H1: Las Autoridades Portuarias que han realizado un mayor esfuerzo en innovación han superado con mayor holgura la crisis,

Posteriormente, Goya et al., (2012) demuestran que la innovación tiene un impacto positivo sobre la productividad, encontrando un mayor efecto en empresas de mayor tamaño y en sectores de alta tecnología, por lo que nos planteamos la segunda hipótesis:

H2: Las Autoridades Portuarias más grandes son las que mayor esfuerzo inversor en innovación realizan.

Jaumandreu y Doraszelski (2007) investigaron la relación entre I+D y productividad en nueve sectores industriales españoles durante la década de los 90, llegando a la conclusión de que la I+D es la variable principal que explica las diferencias de productividad de las empresas. Más recientes son las conclusiones de Naider (2012), quien determinó que la correlación entre la inversión en innovación y las ventas de las empresas podría estar entre un 1,099 y un 1,107.

En el ámbito internacional, Crépon et al., (1998) y Duguet (2006), concluyeron, para distintos periodos y muestras empresariales francesas, que existía una correlación positiva entre la participación en actividades de Investigación y Desarrollo (I+D) y la cuota de mercado, es decir, entre el esfuerzo innovador y las ventas. Ernst (1995) realizó un estudio en Alemania en una muestra de 50 empresas de la industria de ingeniería mecánica con el objetivo de relacionar las patentes ${ }^{\bullet}$ con las ventas en el periodo 1984-1991, utilizando para ello diversos indicadores de patentes y de crecimiento de las ventas. Sus conclusiones muestran que las empresas que solicitan más patentes son aquellas que también tienen mayor crecimiento de las ventas. En un artículo posterior, Ernst (2001) encuentra que la solicitud de patentes nacionales incrementa las ventas en un periodo de tiempo de entre dos y tres años después del año de prioridad de la patente. Además, la solicitud de patentes europeas tiene un impacto aún mayor en el crecimiento de las ventas en un periodo de tiempo de tres años posterior al año de prioridad. Chen (2011) realiza un estudio en la industria farmacéutica estadounidense haciendo uso de diversas variables relacionadas con los aspectos de las patentes y utilizando el porcentaje de crecimiento anual de las ventas como variable dependiente, encontrando una relación positiva entre ambas variables. Por su parte, Griffith et al., (2006) realizan una comparación entre cuatro países europeos (Francia, Alemania, España y Reino Unido) sobre la relación entre innovación y productividad empresarial y concluyen que las innovaciones en producto incrementan la productividad solamente en Francia, España y Reino Unido, no encontrando relación en Alemania. Sin embargo, las innovaciones en proceso están correlacionadas positivamente con la productividad en Francia. Por otra parte, O'Mahoney y Vecchi

\footnotetext{
- Las patentes son solamente una parte de la propiedad industrial, por lo tanto, los trabajos de Ernst $(1995,2001)$ y Chen (2011) han usado únicamente como variable proxy un solo componente de la propiedad industrial.
} 
(2009) encuentran una relación positiva entre productividad y capital en I+D, en empresas de Estados Unidos, Reino Unido, Japón, Francia y Alemania descubriendo, además, que las industrias intensivas en I+D tienden a incrementan su productividad entre un $2 \%$ y un $5 \%$. Por último, Goedhuys (2007), analizó si el crecimiento en las ventas de 1642 empresas brasileñas en el periodo 2000-2002 estaba relacionado con diversas actividades de innovación entre las que consideró: si la empresa formaba parte de una nueva joint-venture o no, si se invertía en I+D o en diseño, si había desarrollado una nueva línea de productos o había mejorado las existentes, si había introducido una nueva tecnología que hubiera mejorado significativamente la producción de un producto, si habían hecho uso de la web o no, si tenían certificación ISO o no, o si, por ejemplo, cooperaba con clientes para adquirir nueva tecnología o no. Sus resultados mostraron que las actividades de innovación explican las diferencias existentes en el crecimiento de las ventas, incluso en sectores tradicionales en los que las empresas brasileñas tienen ventajas competitivas. Por consiguiente, y de acuerdo con la justificación teórica, formulamos nuestra tercera y última hipótesis:

H3: Existe una relación directa y positiva entre inversión en innovación e ingresos de las Autoridades Portuarias.

\section{DESCRIPCIÓN DE VARIABLES Y METODOLOGÍA.}

Para analizar los efectos de la inversión en innovación tecnológica de las AAPP hemos consultado las Memorias de las AAPP desde 2008 a 2015 (Ministerio de Fomento, 2018), y así obtener las Cuentas Anuales de las 28 AAPP. Hemos centrado el análisis del estudio en el periodo 2008-2015 por ser el más actual desde que se introdujo el nuevo Plan General de Contabilidad de empresas 2007 (PGC) y porque considera años de crisis económica: 2008-2012 y años de recuperación económica: 2013-15. La variable proxy de la inversión en innovación ha sido la cuenta 203: propiedad industrial, ya que la propiedad industrial es definida por el PGC de 2007 como "el importe satisfecho por la propiedad o por el derecho al uso o a la concesión del uso de las distintas manifestaciones de la propiedad industrial, en los casos en que, por las estipulaciones del contrato, deban inventariarse por la empresa adquirente. Este concepto incluye, entre otras, las patentes de invención, los certificados de protección de modelos de utilidad pública y las patentes de introducción", lo que siguiendo a Mahnazmoradi (2017) nos podría aproximar a establecer una cuantificación global de la innovación tanto de servicios, como de procesos o como de la mejora administrativa.

Hemos construido un panel de datos en el que se recogen tanto los importes de la cuenta 203: propiedad industrial como las grandes masas patrimoniales de los balances y de la cuenta de resultados de cada Autoridad Portuaria en cada año del periodo estudiado: Activo Total (AT), Activo no Corriente (ANC), Fondos Propios (FP) Pasivo Total (PT), Cifra Neta de Negocios (CNN), Resultado de Explotación (REX).

Posteriormente, y para analizar si el tamaño tenía relación con las decisiones de inversión en innovación, se han clasificado las instituciones por tamaño atendiendo a la media en el periodo de su CNN. De tal modo que, utilizando como guía los límites que establece el Plan General de Contabilidad de 2007 sobre la CNN para la presentación de cuentas de pérdidas y ganancias en modelo normal o modelo abreviado, surgieron tres grupos de instituciones. El primero estaba compuesto por las AAPP que arrojaban una media de la cifra neta de negocios en el periodo superior a $22.800 .000 €$. Fueron 13 instituciones y las denominamos Autoridades Portuarias Grandes (AAPPG). El segundo eran otras 13 Autoridades Portuarias que las denominamos medianas (AAPPM) porque mostraban una media de la $\mathrm{CNN}$ en el periodo inferior a $22.800 .000 €$ pero superior a $8.000 .000 €$. Y, por último, aparecieron 2 Autoridades Portuarias con una media de la CNN inferior a 8 millones de euros que, para establecer nuestro ranking, las llamamos Autoridades Portuarias Pequeñas (AAPPP).

A continuación, y siguiendo a Rivero (1996), Gonzalo et al., (2000); Archel et al., (2008) o Garrido e Íñiguez (2012) se han definido un conjunto de ratios económico-financieras que miden aspectos patrimoniales de las AAPP como son el apalancamiento financiero o la solvencia: Ratio de Solvencia (RS) y de Autonomía Financiera (AF). Y otros que se centran en el análisis de los resultados: Margen de Explotación (MEX), la Rentabilidad Económica (ROA), la Rentabilidad Financiera (ROE). A estas ratios de tipo financiero, y siguiendo a Mahnazmoradi (2017), se les ha añadido otra ratio que pretende evaluar y cuantificar la Intensidad Innovadora (II) de las AAPP. (Tabla 2). 
Tabla 2

Definición de ratios y variables.

\begin{tabular}{|c|c|c|}
\hline \multicolumn{2}{|l|}{ Factor } & Definición \\
\hline \multirow{2}{*}{$\begin{array}{l}\text { Ratios de balance o } \\
\text { equilibrio }\end{array}$} & $\begin{array}{l}\text { Solvencia: } \\
\text { (RS) }\end{array}$ & Activo Total/Pasivo Total (AT/PT) \\
\hline & $\begin{array}{l}\text { Autonomía Financiera: } \\
(\mathrm{AF})\end{array}$ & Fondos Propios/Pasivo Total (FP/PT) \\
\hline \multirow{3}{*}{ Ratios de Resultados } & $\begin{array}{l}\text { Margen de Explotación: } \\
\text { (MEX) }\end{array}$ & $\begin{array}{l}\text { Beneficio Antes de Intereses e Impuestos/Cifra Neta } \\
\text { de Negocios (BAIT/CNN) }\end{array}$ \\
\hline & $\begin{array}{l}\text { Rentabilidad Económica } \\
\text { (ROA) }\end{array}$ & $\begin{array}{l}\text { Beneficio Antes de Intereses e Impuestos/Activo Total } \\
\text { (BAIT/AT) }\end{array}$ \\
\hline & $\begin{array}{l}\text { Rentabilidad Financiera } \\
\text { (ROE) }\end{array}$ & Beneficio Neto/Fondos Propios (BN/FP) \\
\hline Ratios de Innovación & $\begin{array}{l}\text { Intensidad Innovadora } \\
\text { (II) }\end{array}$ & $\begin{array}{l}\text { Inversión en Innovación/Cifra Neta Negocios } \\
\text { (Propiedad Industrial/CNN) }\end{array}$ \\
\hline
\end{tabular}

Elaboración propia.

Para el análisis de las ratios, y por consistencia estadística, hemos establecido solo dos tamaños: las grandes (AAPPG) que son 13 y el grupo de Autoridades Portuarias Pyme (AAPPPyme) en el que hemos englobado las 13 medianas y las 2 pequeñas.

Posteriormente y con SPSS22, hemos analizado los cambios en las medias muestrales de cada una de las 6 ratios seleccionadas desde 2008 hasta 2015 y para cada grupo de nuestras AAPP mediante un contraste de hipótesis bilateral y con un intervalo de confianza del $95 \%$. A través del estadístico F y del p-valor en la prueba Anova, hemos contrastado la hipótesis nula de igualdad de medias para cada ratio en el periodo objeto de estudio y hemos rechazado la hipótesis nula de igualdad de medias para cada ratio que mostraba un p-valor (o significación bilateral) inferior a 0,05. Posteriormente, se ha aplicado un análisis multivariante Post Hoc de Tukey HDS (Honestly-significant-difference) o Manova comparando las variables para cada par de años. Con ello, comprobamos si en los casos donde se ha rechazado la hipótesis nula de igualdad de medias, afecta a todos los pares de años o, por el contrario, solamente afecta a alguno de ellos.

Por último, y para estudiar la relación entre esfuerzo innovador y resultados económicos de cada Autoridad Portuaria, se han elaborado unos coeficientes de correlación de Pearson entre la evolución de la cuenta "propiedad industrial", los resultados empresariales medidos a través de la CNN y la estructura económica y financiera de las AAPP medidas a través de sus grandes masas patrimoniales (AT, FP, ANC, PT).

\section{RESULTADOS}

La tabla 3 muestra el ranking de las AAPP utilizando la media de la CNN para ordenarlas de mayor a menor. En la columna 4, describimos si la institución en cuestión ha realizado inversión en innovación a través de los importes reflejados en la cuenta (203): propiedad industrial de sus balances de situación a 31 de diciembre de cada año en el periodo 2008-2015. 
Tabla 3

Clasificación por tamaño de las Autoridades Portuarias según la media de la CNN del periodo e inversión en Propiedad Industrial.

\begin{tabular}{|c|c|c|c|c|}
\hline $\begin{array}{l}\text { Nombre de la } \\
\text { Autoridad } \\
\text { Portuaria }\end{array}$ & $\begin{array}{l}\text { Posición } \\
\text { en el } \\
\text { Ranking }\end{array}$ & $\begin{array}{l}\text { Clasificación } \\
\text { por tamaño }\end{array}$ & $\begin{array}{l}\text { Inversión en } \\
\text { propiedad } \\
\text { industrial (203) } \\
\text { durante algún año } \\
\text { del periodo }\end{array}$ & $\begin{array}{l}\text { Criterio de } \\
\text { Clasificación }\end{array}$ \\
\hline Barcelona & 1 & GRANDES & Sí & \multirow{13}{*}{ 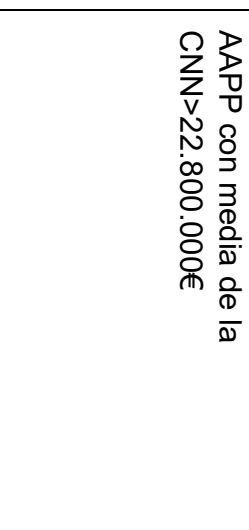 } \\
\hline Valencia & 2 & GRANDES & Sí & \\
\hline Algeciras & 3 & GRANDES & No & \\
\hline Bilbao & 4 & GRANDES & No & \\
\hline Las palmas & 5 & GRANDES & Sí & \\
\hline Baleares & 6 & GRANDES & Sí & \\
\hline Tarragona & 7 & GRANDES & Sí & \\
\hline Huelva & 8 & GRANDES & Sí & \\
\hline Cartagena & 9 & GRANDES & Sí & \\
\hline Gijón & 10 & GRANDES & Sí & \\
\hline Tenerife & 11 & GRANDES & No & \\
\hline Vigo & 12 & GRANDES & Sí & \\
\hline La Coruña & 13 & GRANDES & Sí & \\
\hline Castellón & 14 & MEDIANAS & No & \multirow{13}{*}{ 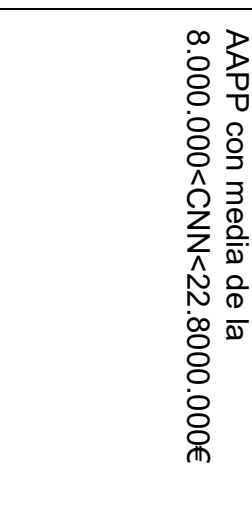 } \\
\hline Santander & 15 & MEDIANAS & No & \\
\hline Cádiz & 16 & MEDIANAS & Sí & \\
\hline Sevilla & 17 & MEDIANAS & No & \\
\hline Ferrol & 18 & MEDIANAS & Sí & \\
\hline Málaga & 19 & MEDIANAS & Sí & \\
\hline Ceuta & 20 & MEDIANAS & No & \\
\hline Pasajes & 21 & MEDIANAS & No & \\
\hline Avilés & 22 & MEDIANAS & Sí & \\
\hline Almería & 23 & MEDIANAS & Sí & \\
\hline Alicante & 24 & MEDIANAS & No & \\
\hline Melilla & 25 & MEDIANAS & No & \\
\hline Pontevedra & 26 & MEDIANAS & No & \\
\hline Motril & 27 & PEQUEÑAS & Sí & \multirow{2}{*}{$\begin{array}{l}\text { AAPP con media } \\
\text { de la } \\
\text { CNN }<8.000 .000 €\end{array}$} \\
\hline Villa Arosa & 28 & PEQUEÑAS & Sí & \\
\hline
\end{tabular}

Elaboración propia

De su observación comprobamos que no todas las AAPP han invertido en propiedad industrial y, por tanto, en innovación. De las 28 solo 17 han realizado algún tipo de innovación ya sea de prototipos, de procesos, administrativo o de marca. Además, aunque en su mayoría coinciden con los grandes puertos, llama la atención que ni el de Algeciras, ni el de Bilbao ni el de Tenerife siendo de los más grandes en España, sobre todo, el puerto de Algeciras y el de Bilbao que ocupan la posición tercera y cuarta respectivamente, ninguno ha invertido en propiedad industrial durante el periodo analizado. Por el contrario, puertos muy pequeños y modestos como el de Motril o el de Villa de Arosa, mantienen un esfuerzo inversor en innovación por encima de la media.

La tabla 4 representa los estadísticos descriptivos de las ratios económico-financieros y de innovación de las autoridades portuarias. 
Tabla 4

Estadísticos descriptivos de las ratios económico-financieros e intensidad en innovación de las Autoridades Portuarias por tamaño.

\begin{tabular}{|c|c|c|c|c|c|c|c|c|c|c|c|c|c|}
\hline & \multirow[t]{2}{*}{ Desc } & \multicolumn{6}{|l|}{ AAPPG } & \multicolumn{6}{|c|}{ AAPPPyme } \\
\hline & & MEX & ROA & ROE & RS & AF & II & MEX & ROA & ROE & RS & AF & II \\
\hline \multirow[t]{4}{*}{2008} & Media & 0,33 & 0,03 & 0,03 & 10,24 & 9,24 & 0,01 & 0,37 & 0,03 & 0,02 & 11,26 & 10,26 & 0,00 \\
\hline & Desv. típ. & 0,12 & 0,02 & 0,02 & 9,03 & 9,03 & 0,02 & 0,57 & 0,05 & 0,02 & 14,31 & 14,31 & 0,01 \\
\hline & Mín & 0,06 & 0,00 & 0,00 & 2,45 & 1,45 & 0,00 & $-0,18$ & $-0,01$ & $-0,04$ & 2,85 & 1,85 & 0,00 \\
\hline & Máx & 0,48 & 0,05 & 0,07 & 27,73 & 26,73 & 0,07 & 2,25 & 0,20 & 0,05 & 54,41 & 53,41 & 0,05 \\
\hline \multirow[t]{4}{*}{2009} & Media & 0,08 & 0,01 & 0,00 & 6,33 & 5,33 & 0,01 & $-0,16$ & $-0,01$ & $-0,03$ & 6,76 & 5,76 & 0,00 \\
\hline & Desv. típ. & 0,33 & 0,03 & 0,05 & 6,36 & 6,36 & 0,02 & 0,38 & 0,04 & 0,08 & 6,85 & 6,85 & 0,01 \\
\hline & Mín & $-0,67$ & $-0,07$ & $-0,12$ & 1,95 & 0,95 & 0,00 & $-1,05$ & $-0,12$ & $-0,30$ & 2,42 & 1,42 & 0,00 \\
\hline & Máx & 0,50 & 0,05 & 0,05 & 24,29 & 23,29 & 0,07 & 0,32 & 0,02 & 0,03 & 28,23 & 27,23 & 0,03 \\
\hline \multirow[t]{4}{*}{2010} & Media & 0,37 & 0,03 & 0,04 & 7,45 & 6,45 & 0,01 & 0,17 & 0,01 & 0,02 & 6,00 & 5,00 & 0,00 \\
\hline & Desv. típ. & 0,31 & 0,03 & 0,05 & 6,88 & 6,88 & 0,02 & 0,28 & 0,02 & 0,03 & 4,80 & 4,80 & 0,01 \\
\hline & Mín & 0,04 & 0,00 & $-0,02$ & 1,74 & 0,74 & 0,00 & $-0,28$ & $-0,01$ & $-0,02$ & 2,26 & 1,26 & 0,00 \\
\hline & Máx & 1,15 & 0,11 & 0,19 & 21,62 & 20,62 & 0,06 & 0,90 & 0,07 & 0,10 & 20,82 & 19,82 & 0,04 \\
\hline \multirow[t]{4}{*}{2011} & Med & 0,23 & 0,02 & 0,02 & 10,45 & 9,45 & 0,01 & 0,13 & 0,01 & 0,01 & 7,66 & 6,66 & 0,00 \\
\hline & Desv. típ. & 0,15 & 0,01 & 0,02 & 11,12 & 11,12 & 0,02 & 0,14 & 0,01 & 0,02 & 6,27 & 6,27 & 0,01 \\
\hline & Mín & $-0,02$ & 0,00 & $-0,02$ & 1,70 & 0,70 & 0,00 & $-0,11$ & $-0,01$ & $-0,02$ & 2,24 & 1,24 & 0,00 \\
\hline & Máx & 0,48 & 0,04 & 0,05 & 34,18 & 33,18 & 0,06 & 0,33 & 0,03 & 0,04 & 21,16 & 20,16 & 0,04 \\
\hline \multirow[t]{4}{*}{2012} & Media & 0,22 & 0,02 & 0,02 & 15,21 & 14,21 & 0,01 & 0,14 & 0,01 & 0,01 & 9,88 & 8,88 & 0,00 \\
\hline & Desv. típ. & 0,18 & 0,02 & 0,02 & 18,41 & 18,41 & 0,03 & 0,12 & 0,01 & 0,01 & 11,70 & 11,70 & 0,01 \\
\hline & Mín & $-0,14$ & 0,00 & $-0,01$ & 1,99 & 0,99 & 0,00 & 0,01 & 0,00 & $-0,02$ & 2,28 & 1,28 & 0,00 \\
\hline & Máx & 0,56 & 0,06 & 0,07 & 58,63 & 57,63 & 0,13 & 0,39 & 0,03 & 0,03 & 46,55 & 45,55 & 0,04 \\
\hline \multirow[t]{4}{*}{2013} & Media & 0,21 & 0,02 & 0,02 & 15,00 & 14,00 & 0,01 & 0,09 & 0,01 & 0,01 & 16,35 & 15,35 & 0,00 \\
\hline & Desv. típ. & 0,17 & 0,01 & 0,02 & 19,69 & 19,69 & 0,03 & 0,15 & 0,01 & 0,01 & 28,82 & 28,82 & 0,01 \\
\hline & Mín & $-0,16$ & 0,00 & $-0,01$ & 2,11 & 1,11 & 0,00 & $-0,27$ & $-0,01$ & $-0,02$ & 2,42 & 1,42 & 0,00 \\
\hline & Máx & 0,45 & 0,04 & 0,05 & 72,48 & 71,48 & 0,12 & 0,31 & 0,03 & 0,03 & 111,34 & 110,34 & 0,04 \\
\hline \multirow[t]{4}{*}{2014} & Media & 0,22 & 0,02 & 0,02 & 16,17 & 15,17 & 0,02 & 0,16 & 0,01 & 0,02 & 18,59 & 17,59 & 0,01 \\
\hline & Desv. típ. & 0,14 & 0,01 & 0,02 & 22,16 & 22,16 & 0,06 & 0,18 & 0,02 & 0,03 & 34,85 & 34,85 & 0,01 \\
\hline & Mín & 0,03 & 0,00 & $-0,01$ & 2,07 & 1,07 & 0,00 & $-0,15$ & $-0,01$ & $-0,01$ & 2,51 & 1,51 & 0,00 \\
\hline & Máx & 0,52 & 0,05 & 0,05 & 80,38 & 79,38 & 0,23 & 0,53 & 0,06 & 0,13 & 133,23 & 132,23 & 0,04 \\
\hline \multirow[t]{4}{*}{2015} & Media & 0,21 & 0,02 & 0,02 & 15,93 & 14,93 & 0,02 & 0,12 & 0,01 & 0,02 & 21,50 & 20,50 & 0,01 \\
\hline & Desv. típ. & 0,12 & 0,01 & 0,02 & 19,69 & 19,69 & 0,06 & 0,17 & 0,02 & 0,03 & 30,86 & 30,86 & 0,01 \\
\hline & Mín & $-0,03$ & 0,00 & 0,00 & 2,17 & 1,17 & 0,00 & $-0,13$ & 0,00 & $-0,01$ & 2,68 & 1,68 & 0,00 \\
\hline & Máx & 0,45 & 0,04 & 0,04 & 57,20 & 56,20 & 0,20 & 0,47 & 0,06 & 0,13 & 108,68 & 107,68 & 0,04 \\
\hline
\end{tabular}

Elaboración Propia.

De la observación de los estadísticos se desprenden cuatro ideas básicas.

En primer lugar, el año 2009 fue un año nefasto para todas las AAPP, el margen de explotación (MEX) disminuyó considerablemente, pasando de ofrecer unos holgados resultados positivos a convertirse en negativos, sobre todo para el grupo de las APPPyme. La crisis económica internacional y su consecuente repercusión en el tráfico marítimo internacional fueron los desencadenantes de este comportamiento.

El año 2010, parecía ser un año de recuperación para el sector del comercio internacional y, por tanto, de crecimiento del tráfico portuario español, sin embargo, el grado de cobertura de los ingresos de explotación frente a los gastos nunca alcanzó el nivel de antes de la crisis y, por consiguiente, el MEX en 2015 no llegó a ser el 65\% de lo que fue el MEX durante el año 2008.

Mayor aun fue la caída del MEX para el caso de las AAPPPyme, ya que pasaron de tener una rentabilidad antes de impuestos e intereses superior a la de las AAPPG a estar casi a la mitad en 2015 $\mathrm{y}$, por tanto, no llegar a tener el $35 \%$ del margen o rentabilidad de negocio que obtuvieron durante en 2008. 
Los estadísticos también describen que el grupo de las AAPPPyme tienen una menor intensidad investigadora (II), lo que unido a la mayor pérdida de rentabilidad de negocio y la menor capacidad de recuperación durante el periodo de crecimiento económico, estaría en consonancia con las conclusiones de Somohano et al., (2018), quienes confirmaron que en el contexto de crisis económica las empresas que realizaron menos inversión en innovación tuvieron peores resultados económicos, ya que el hecho de que una empresa tenga propiedad industrial le concede un monopolio durante un determinado periodo de tiempo que impide a sus competidores la fabricación y venta de su producto o servicio o incluso imposibilita la utilización de un proceso.

La propiedad industrial permite que la empresa propietaria disfrute, por un lado, de una exclusividad de uso, de gestión o de producción y venta de servicios, de productos o de procesos y, de otro lado, faculta a la empresa para obtener abultados márgenes, ya que la barrera de entrada en la industria a la competencia le crea una ventaja competitiva que, para el caso de patentes, llega a los 20 años. Por tanto, en general, las empresas que mantienen una inversión en propiedad industrial pueden reducir el riesgo por disminución de resultados empresariales, sobre todo en épocas de recesión.

En segundo lugar, y como posible consecuencia de la mejora del MEX, se aprecia que desde 2010 la rentabilidad de las instituciones se ha ido recuperando paulatinamente, sobre todo en las AAPPG, que sin llegar a alcanzar la rentabilidad económica (ROA) y la rentabilidad financiera (ROE) del periodo pre-crisis, ya en los últimos años muestran una rápida y sólida recuperación. Pero a pesar de la recuperación económica, la dificultad para alcanzar una rentabilidad de los activos del 3\% (como la que se ofreció en 2008) y la lentitud en la recuperación de la ROA y la ROE en el caso de las AAPPyme, nos pueden estar indicando que el crecimiento del comercio internacional desde $2010 \mathrm{y}$, por consiguiente, el aumento del tráfico portuario español a partir de 2010 (Tabla 1) no han sido capaces de proporcionar una auténtica recuperación de la rentabilidad económica a la gestión portuaria española. Por todo ello y para nuestro estudio, podemos estar en disposición de aceptar nuestra primera hipótesis: H1: Las Autoridades Portuarias que han realizado un mayor esfuerzo en innovación han superado con mayor holgura la crisis y es posible que el modelo de negocio esté cambiando y sea necesario una mayor inversión en innovación y modernización tanto de los servicios que se ofrecen para el tradicional tráfico portuario de mercancías como de la oferta de nuevos servicios y actividades que puedan generar mayor rentabilidad.

En tercer lugar, las ratios de balance y equilibrio: ratio de solvencia (RS) y ratio de autonomía financiera $(\mathrm{AF})$ muestran una alta capacidad para hacer frente a las deudas con recursos propios y un bajo grado de apalancamiento de todas las AAPP. El escaso endeudamiento y el alto volumen de recursos propios que, se incrementa durante la fase de recuperación económica, sobre todo para el grupo de pequeños y medianos puertos que lo duplican, nos hacen pensar que los puertos españoles podrían estar mejor gestionados y ser más rentables, ya que siguiendo a Garrido e Íñiguez (2012), el exceso de recursos propios ociosos no es óptimo porque por un lado, perjudica a los propietarios al tener que soportar todo el riesgo financiero de la empresa y por otro, tiene un coste de oportunidad al no invertir en mejoras tanto de instalaciones como de proyectos como de servicios, lo que traería una mejora de los resultados económicos por un posible aumento de la competitividad portuaria.

Por último, la evolución de la ratio intensidad innovadora (II) sugiere que son las Autoridades Portuarias más grandes las que más invierten en propiedad industrial, lo que viene a corroborar la Tabla 3 y está en consonancia con las conclusiones de Buesa y Molero (1998), puesto que, según éstos autores, cuanto mayor sea la dimensión de la empresa, más actividades relacionadas con la innovación realizarán: las empresas que más venden, más se modernizan.

Por ello y para nuestro estudio, podemos estar en disposición de aceptar nuestra segunda hipótesis: H2: Las Autoridades Portuarias más grandes son las que mayor esfuerzo inversor en innovación realizan.

En la tabla 5 se muestran los resultados del análisis Anova, con el objetivo de examinar si la media de cada ratio difiere o no de manera significativa a lo largo de la crisis. Se rechaza la hipótesis nula de igualdad de medias con un intervalo de confianza del $95 \%$ en todas aquellas ratios que alcanzan un pvalor $<0,05$. Lo que posteriormente ha corroborado con el análisis Post Hoc de Tukey HDS (Honestlysignificant-difference) o Manova, en el que se ha comparado el valor de cada ratio de dos años en dos años para comprobar si los casos en donde se ha rechazado la hipótesis nula de igualdad de medias, afectan a todos los pares de años o sólo a alguno. 
Tabla 5

Contrastes de hipótesis y análisis de la varianza Anova.

\begin{tabular}{|l|r|r|r|r|r|r|}
\hline & \multicolumn{1}{|l|}{ AAPPG } & \multicolumn{1}{|l|}{ AAPPPyme } & \multicolumn{2}{l|}{ TOTAL AAPP } \\
\hline & $\mathrm{F}$ & Sig (p-valor). & $\mathrm{F}$ & Sig (p-valor) & $\mathrm{F}$ & Sig (p-valor) \\
\hline MEX & 2,21 & 0,04 & 3,77 & 0,00 & 5,40 & 0,00 \\
\hline ROA & 1,87 & 0,04 & 3,30 & 0,00 & 4,85 & 0,00 \\
\hline ROE & 2,18 & 0,04 & 3,39 & 0,00 & 4,70 & 0,00 \\
\hline RS & 0,86 & 0,54 & 1,19 & 0,32 & 2,04 & 0,05 \\
\hline AF & 0,86 & 0,54 & 1,19 & 0,32 & 2,04 & 0,05 \\
\hline II & 0,44 & 0,87 & 0,28 & 0,96 & 0,63 & 0,73 \\
\hline
\end{tabular}

Elaboración propia.

Atendiendo al estadístico F y al p-valor surgido del análisis Anova, se puede deducir que han existido cambios estadísticamente significativos en las ratios relacionadas con los resultados económicos de las autoridades portuarias (MEX, ROA, ROE), tanto en las medianas y pequeñas, como en las grandes, y para el total de las AAPP españolas.

En efecto, la fuerte caída del MEX 2009 fue rápidamente superada. Sin embargo, ni las grandes y, mucho menos las AAPP más pequeñas han sido capaces de alcanzar un nivel de cobertura de sus gastos de explotación tan alto como el del periodo pre-crisis, ya que ahora necesitan obtener más ingresos para conseguir los mismos resultados.

El incremento del tráfico portuario no ha sido suficiente acicate para que se recuperase la CNN y la falta de un aumento de los ingresos por otros conceptos unido al mantenimiento de una estructura de gastos de explotación cuasi-fijos, fundamentalmente en el caso de las AAPPPyme, conlleva a que el MEX a duras penas alcance la mitad de lo que fue antes de la crisis. Lo que nos sugiere que el modelo de negocio basado exclusivamente en un gran movimiento de contenedores y mercancías se puede estar agotando. La crisis y su superación nos han trasladado a un nuevo contexto económico internacional donde es imprescindible modernizar y transformar los tipos y las ofertas de negocio. Donde la innovación y mejora tanto en la calidad de la gestión administrativa, como el tratamiento de los procesos, como, sobre todo, en el tipo y calidad de servicios que se oferten se pueden estar convirtiendo en piezas claves fundamentales para conseguir un crecimiento sostenible de los resultados económicos de los puertos españoles.

La recuperación parcial del MEX ha afectado en el mismo sentido a la rentabilidad de los activos (ROA). Los puertos españoles aún no han alcanzado los niveles de rentabilidad perdidos por la crisis y la caída del comercio y del tráfico marítimo internacional. Sus activos son ahora menos rentables, lo que abunda en la idea de que un cambio en el modelo de negocio que venga acompañado de una diversificación de actividades y unas mejoras cualitativas de la oferta existente podría ser el sustento para mantener, incluso para aumentar, la cuota de mercado y la competitividad.

Desde este punto de vista, llama la atención que, aunque no hayan existido cambios estadísticamente significativos en la intensidad innovadora (II), sí que se ha producido un crecimiento considerable, sobre todo, para el periodo de recuperación económica. En este sentido, todas las AAPP, independientemente del tamaño, parece que hayan hecho suyas las conclusiones científicas que apuntan a que la inversión en innovación mejora los resultados y la competitividad. Ahora bien, la reinversión de los beneficios en intangibles como la propiedad industrial no ha sido de igual intensidad en los dos grupos de AAPP. Las grandes vienen realizando un esfuerzo inversor en innovación desde antes de que se iniciara la crisis, y que, en los últimos años, se ha duplicado pasando a suponer algo más del $2 \%$ de sus ventas durante 2015. Por el contrario, la reinversión en el caso de las AAPPPyme solo alcanza el $1 \%$ de sus ventas y únicamente para la sub-etapa de recuperación económica, lo que nos ratifica en la aceptación de nuestra segunda hipótesis y nos permite aceptar nuestra tercera hipótesis H3: Existe una relación directa y positiva entre inversión en innovación e ingresos de las Autoridades Portuarias.

La relación entre las ventas, los resultados empresariales y la reinversión de beneficios en actividades de innovación se presenta en la Tabla 6, donde se muestran los coeficientes de correlación de Pearson entre la evolución de la inversión en innovación medida a través de la cuenta (203) propiedad industrial sobre los resultados empresariales y masas patrimoniales. 
Tabla 6

Coeficientes de correlación de Pearson de la innovación sobre masas patrimoniales y resultados empresariales. Autoridades Portuarias Grandes (AAPPG) y Autoridades Portuarias Medianas y Pequeñas (AAPPPyme).

\begin{tabular}{|l|l|l|l|l|l|l|l|}
\hline & $\begin{array}{l}\text { Propiedad } \\
\text { Industrial (PI) }\end{array}$ & $\begin{array}{l}\text { Activo } \\
\text { Total } \\
\text { (AT) }\end{array}$ & $\begin{array}{l}\text { Activo no } \\
\text { Corriente } \\
\text { (ANC) }\end{array}$ & $\begin{array}{l}\text { Fondos } \\
\text { Propios } \\
\text { (FP) }\end{array}$ & $\begin{array}{l}\text { Pasivo } \\
\text { Total } \\
\text { (PT) }\end{array}$ & $\begin{array}{l}\text { Cifra Neta } \\
\text { de } \\
\text { Negocios } \\
\text { (CNN) }\end{array}$ & $\begin{array}{l}\text { Resultado de } \\
\text { Explotación } \\
\text { (REX) }\end{array}$ \\
\hline $\begin{array}{l}\text { Propiedad } \\
\text { Industrial (PI) }\end{array}$ & & $0,69^{* *}$ & $0,67^{* *}$ & $0,70^{* *}$ & $0,51^{* *}$ & $0,66^{* *}$ & $0,56^{* *}$ \\
\hline $\begin{array}{l}\text { Activo } \\
\text { Total (AT) }\end{array}$ & 0,02 & & $0,98^{* *}$ & $0,90^{* *}$ & $0,88^{* *}$ & $0,86^{* *}$ & $0,56^{* *}$ \\
\hline $\begin{array}{l}\text { Activo no } \\
\text { Corriente } \\
\text { (ANC) }\end{array}$ & 0,01 & $0,99^{* *}$ & & $0,89^{* *}$ & $0,86^{* *}$ & $0,85^{* *}$ & $0,53^{* *}$ \\
\hline $\begin{array}{l}\text { Fondos (FP) } \\
\text { Propios (FP) }\end{array}$ & 0,03 & $0,95^{* *}$ & $0,94^{* *}$ & & $0,60^{* *}$ & $0,88^{* *}$ & $0,60^{* *}$ \\
\hline $\begin{array}{l}\text { Pasivo Total } \\
\text { (PT) }\end{array}$ & 0,01 & $0,66^{* *}$ & $0,68^{* *}$ & $0,41^{* *}$ & & 060 & 0,36 \\
\hline $\begin{array}{l}\text { Cifra Neta de } \\
\text { Negocios } \\
\text { (CNN) }\end{array}$ & 0,03 & $0,74^{* *}$ & $0,74^{* *}$ & $0,69^{* *}$ & $0,54^{* *}$ & & $0,70^{* *}$ \\
\hline $\begin{array}{l}\text { Resultado de } \\
\text { Explotación } \\
\text { (REX) }\end{array}$ & 0,04 & 0,14 & 0,13 & 0,14 & 0,06 & $0,36^{* *}$ & \\
\hline
\end{tabular}

Elaboración propia.

Clave: En la parte de la tabla situada por encima de la diagonal principal aparecen los valores del coeficiente de correlación de Pearson para las masas patrimoniales y resultados de las AAPP Grandes (AAPPG). En la parte situada por debajo aparecen los correspondientes valores del mismo coeficiente Pearson para las masas patrimoniales y resultados de las AAPP Medianas y Pequeñas (AAPPPyme).

$\left.{ }^{* *}\right)$ La correlación es significativa bilateral al nivel 0,01 .

El valor del coeficiente de correlación varía desde -1 a +1 , donde 0 significa que no existe relación entre las variables. Por tanto, al observar la relación entre las variables propiedad industrial (PI) y cifra neta de negocios $(\mathrm{CNN})$, se comprueba que el coeficiente de correlación es positivo de 0,66 con un nivel de significación de 0,01 para las (AAPPG) (cuadrante superior de la diagonal) mientras que para las AAPPPyme el coeficiente es de 0,03. Esta escasa correlación entre propiedad industrial e ingresos por ventas vendría razonada por el hecho de que más de la mitad de las AAPPPyme no han tenido durante todo el periodo objeto de estudio inversión en propiedad industrial y, las que en algún año la tuvieron, terminaron de amortizarla antes de iniciarse la recuperación, no realizando ninguna inversión en $\mathrm{I}+\mathrm{D}$ posteriormente. Esto viene a corroborar nuestra primera idea de que los puertos más grandes, con mayor capacidad de crecimiento y más expuestos a la competencia internacional son los primeros en invertir en intangibles como nuevos prototipos o nuevos procesos como receta para superar la crisis. Ahora bien, la sólida autonomía financiera y de estructura de capital de las Autoridades Portuarias, tanto en las grandes como en las pequeñas y medianas, podría favorecer esta apuesta hacia la inversión en innovación, lo que favorecería la tan deseada mejora de la productividad y competitividad.

\section{CONCLUSIONES}

La innovación es un factor clave en el desarrollo tanto de las regiones como de las empresas. En el presente trabajo, se ha realizado un análisis de cuentas anuales de las Autoridades Portuarias españolas para el periodo 2008-2015 buscando la relación entre innovación, medida a través de la cuenta (203) propiedad industrial, y resultados empresariales.

De análisis de las ratios económicos-financieros se observa que hubo cambios estadísticamente significativos en las medias muestrales tanto del margen de explotación (MEX) como de la rentabilidad de los activos (ROA) y de los fondos propios (ROE). Sobre todo, y como nos señala el análisis Post Hoc de Tukey HDS, los cambios significativos se produjeron durante los primeros años de nuestro periodo de estudio. Esto nos permite afirmar que la crisis afectó rápida y profundamente a la rentabilidad de todas las AAPP, lo que hizo que el año 2009 diera los peores resultados del todo el periodo. Pero la rápida recuperación en el comercio internacional permitió aumentar el tráfico portuario y desde 2010 se invirtió la tendencia de los márgenes y la rentabilidad de activos de los puertos españoles.

Sin embargo, la recuperación no ha sido tan intensa como rápida, ya que durante el 2015 ningún puerto había recuperado la rentabilidad de negocio del periodo pre-crisis. El MEX de las AAPPG solo se había recuperado en un 65\% y el de las AAPPPyme no alcanzaba una recuperación del 35\%, de ahí 
que después de la crisis, los puertos necesitaran vender más para obtener los mismos beneficios que antes. Sobre todo, en el caso de las AAPPyme que precisamente coinciden con el grupo de puertos que mostraba una ratio de intensidad innovadora (II) nulo o casi nulo, lo que va en sintonía con Grandón y Rodríguez (1992) y da validez para aceptar nuestra primera hipótesis H1: Las Autoridades Portuarias que han realizado un mayor esfuerzo en innovación han superado con mayor holgura la crisis. La crisis económica ha cambiado el paradigma del negocio portuario y es necesario modernizar la actividad de los puertos españoles en aras de recuperar la rentabilidad perdida.

Desde este punto de vista, hemos verificado que las autoridades portuarias más grandes son las que han sido capaces de recuperar mejor los márgenes de beneficio y rentabilidad, pero, además, son el grupo que, a excepción de Algeciras y Bilbao, mantienen la cuenta (203) "propiedad industrial" positiva en el periodo. Lo que abunda en la aceptación de nuestra hipótesis (H1) y nos permite aceptar nuestra segunda hipótesis H2: Las Autoridades Portuarias más grandes son las que mayor esfuerzo inversor en innovación realizan.

En este sentido, hemos comprobado que los coeficientes de correlación de Pearson entre innovación, medida como propiedad industrial, y la cifra neta de negocios, son distintos de cero, positivos y próximos a 1, lo que muestra que existe relación entre innovación y resultados, como concluyeron O’Mahony y Vecchi (2009) o Chen (2011), lo que nos lleva a aceptar la hipótesis H3: Existe una relación directa y positiva entre inversión en innovación e ingresos de las Autoridades Portuarias. No obstante, esta relación se da sólo para el caso de las grandes Autoridades Portuarias, lo que está en consonancia con el trabajo de Goya et al., (2012) y con la hipótesis H2: Las Autoridades Portuarias más grandes son las que mayor esfuerzo inversor en innovación realizan.

En conclusión, podemos decir que los puertos más grandes, con mayor capacidad de crecimiento y más expuestos a la competencia internacional son los primeros en invertir en intangibles como nuevos prototipos o nuevos procesos como receta para superar la crisis. Y que esta inversión, que en definitiva queda reflejada en la cuenta (203) "propiedad industrial", permite que el puerto mejore su competitividad, obtenga más beneficios y acabe entrando en el círculo virtuoso que genera la inversión en innovación: cuanto mayor es la inversión en innovación, mejores resultados se obtienen y más se crece y, al crecer más, se opta a ejercer una posición dominante en el mercado y, por tanto, a ganar aún más mercado.

A la luz de estas evidencias, creemos que el presente trabajo aporta información relevante sobre un sector económico, que contribuye significativamente a la creación del PIB y a la generación de empleo. Ahora bien, estos resultados podrían estar estrechamente ligados tanto al tratamiento estadístico utilizado como al horizonte temporal y al país. Pensamos que la comparación con las Autoridades Portuarias de otros países, la ampliación del periodo y sobre todo, la aplicación de nuevas herramientas estadísticas podrían arrojar resultados bastante diferenciadores a la par que interesantes como posible futuro objeto de estudio.

\section{REFERENCIAS BIBLIOGRÁFICAS}

ACOSTA, M.; CORONADO, D. y CERBÁN, M.M. (2007). "Port competitiveness in container traffic from an internal point of view: the experience of the Port of Algeciras Bay". Maritime Policy \& Management, 34(5), pp. 501-520. https://doi.org/10.1080/03088830701585381

ACOSTA, M.; CORONADO, D. y CERBÁN, M.M. (2011). "The Economic Impact of the Port of Tarifa in 2007 and the Forescast for 2015". International Journal of Transport Economics, 38(3), pp. 243-264.

AMAT SALAS, O. (2000). Análisis de estados financieros: fundamentos y aplicaciones. Barcelona: Gestión 2000.

ARCHEL DOMENCH, P.; LIZÁRRAGA DALLO, F. y SÁNCHEZ ALEGRÍA, S. (2008). Estados contables. Elaboración, análisis e interpretación, Madrid: Pirámide.

ARTAL-TUR, A.; GÓMEZ-FUSTER, J.M.; NAVARRO-AZORÍN, J.M. y RAMOS-PARREÑO, J.M. (2015). "Estimating the economic impact of a port through regional input-ouput tables: Case study of the Port of Cartagena (Spain)". Maritime Economics \& Logistics, 18(4), pp. 371-390.

BUESA BLANCO, M. y MOLERO ZAYAS, J. (1998). "Tamaño empresarial e innovación tecnológica en la economía española”. Información Comercial Española, 773, pp. 155-173.

CASTILLO MANZANO J.I. (2001). El puerto Bahía de Algeciras, el motor económico del sur, Autoridad Portuaria de Algeciras: Puertos del Estado.

CHEN, Y. S. (2011). "Using patent analysis to explore corporate growth". Scientometrics, Vol. 88, pp. 433-448.

COTO-MILLÁN, P.; MATEO-MANTECÓN, I. y VILLAVERDE, J. (2010). "The Economic Impact of Ports: Its Importance for the Region and Also the Hinterland". Essays on Port Economics, pp. 167-200. Heidelberg: Physica. 
CRÉPON, B.; DUGUET, E. y MAIRESSEC, J. (1998). "Research, Innovation and Productivity: an econometric analysis at the firm level". Economics of Innovation and new Technology, 7(2), pp. 115-158.

CROSSAN, M. M. y APAYDIN, M. (2010). "A multi-dimensional framework of organizational innovation: A systematic review of the literature". Journal of Management Studies, 47(6), pp. 1154-1191.

DOOMS, M.; HAEZENDONCK, E. y VERBEKE, A. (2010). "Socioeconomic Impact of Ports: Development of a European Port Economic Impact Toolkit 2010". Annual Conference of the International Association of Maritime Economics, Lisboa, 7-9 July 2010.

DUGUET, E. (2006). "Innovation height, spillovers and TFP growth at the firm level: Evidence from French manufacturing". Economics of Innovation and New technology, 15(4-5), pp. 415-442.

ERNST, H. (1995). "Patenting strategies in the German mechanical engineering industry and their relationship to firm performance". Technovation, Vol. 15(4), pp. 225-240.

ERNST, H. (2001). "Patent applications and subsequent changes of performance: evidence from time-series cross-section analyses on the firm level". Research Policy, Vol. 30, pp. 143-157.

FRANZONI, C. y SCELLATO, G. (2011). "Academic patenting and the consequences for scientific research". Australian Economic Review, 44(1), pp. 95-101.

FREEMAN, C. (1994). "The economics of technical change". Cambridge Journal of Economics, 18(5), pp. 463514.

GONZALO ANGULO, J.A., PÉREZ GARCÍA, J. y SERRANO GARCÍA, R. (2000). Contabilidad Superior. Análisis Económico-Financiero y Patrimonial. Madrid: Instituto de Auditores-Censores Jurados de Cuentas de España.

GARRIDO MIRALLES, P. e ÍÑIGUEZ SÁNCHEZ, R. (2012). Análisis de Estados Contables. Elaboración e interpretación de la información financiera, Madrid: Pirámide.

GODOY LÓPEZ, L. (2004). Preparación y análisis de estados financieros. Bilbao: Desclée de Brouwer.

GOEDHUYS, M. (2007). The impact of innovation activities on productivity and firm growth: evidence from Brazil. United Nations University, Maastricht Economic and social Research and training centre on Innovation and Technology.

GONZALO ANGULO, J.A.; PÉREZ GARCÍA, J. y SERRANO GARCÍA, R. (2000). Contabilidad Superior. Análisis Económico-Financiero y Patrimonial, Madrid: Instituto de Auditores-Censores Jurados de Cuentas de España.

GOYA, E.; VAYÁ, E. y SURIÑACH, J. (2012). "Productividad, Innovación y Externalidades sectoriales. Evidencia para España" en XV Encuentro de Economía Aplicada, A Coruña, 7 y 8 de Junio de 2012.

GRANDÓN, V. y RODRÍGUEZ ROMERO, L. (1992). "Capital tecnológico e incrementos de productividad en la industria española (1975-1981)”. En García Delgado, J.L. y Serrano Sanz, J.M. (Coord): Economía española, cultura y sociedad: homenaje a Juan Velarde Fuertes ofrecido por la Universidad Complutense, pp, 857-870. Madrid: EUDEMA Universidad.

GRIFFITH, R.; HUERGO, E.; MAIRESSE, J. y PETERS, B. (2006). "Innovation and productivity across four European countries". Oxford Review of Economic Policy, 22(4), pp. 483-498.

GRILICHES, Z. (1998). R\&D and productivity: the econometric evidence, National Bureau of Economic Research Monography. Chicago: University of Chicago press

GRILICHES, Z. (1990). "Patent statistics as economic indicators: A survey". Journal of Economic Literature, Vol 28(4), pp. 1661-1707.

HARHOFF, D., SCHERER, F. y VOPEL, K. (2003). "Citations, family size, opposition and the value of patent rights". Research Policy, Vol. 32, pp. 1343-1363.

JAUMANDREU, J. y DORASZELSKI, U. (2007). R\&D and productivity: estimating production functions when productivity is endogenous (No. we078652). Universidad Carlos III de Madrid. Departamento de Economía.

KEYNES, J. M. (1998). Teoría general del empleo, el interés y el dinero [1936]. Madrid: Ediciones Aosta.

LANJOUW, J.O. y SCHANKERMAN, M. (2004). "Patent quality and research productivity: measuring innovation with multiple indicators". The Economic Journal, Vol, 114 (april) pp. 441-465.

LEYES DEL ESTADO ESPAÑOL (2011). Real Decreto Legislativo 2/2011, de 5 de septiembre, por el que se aprueba el Texto Refundido de la Ley de Puertos del Estado y de la Marina Mercante. Boletín Oficial del Estado, núm 253, de 20 de octubre de 2011.

LEY DE PATRIMONIO DE LAS ADMINISTRACIONES PÚBLICAS (2003). Ley 33/2003, de 3 de noviembre, del Patrimonio de las Administraciones Públicas. Boletín Oficial del Estado, núm. 264, de 4 de noviembre de 2003.

LUCAS, R. (1988). "On the mechanics of economic development". Journal of Monetary Economics, 22(1): pp. 342.

MAHNAZMORADI (2017). "Investigating the Effect of Innovation on Effective Ways of Increasing Sales: Case Study of Dana Insurance". International Journal of Advanced Biotechnology and Research, 8(3): pp. 20922099

MEMORIAS DE LAS AUTORIDADES PORTUARIAS (2008-2016). Puertos del Estado. Madrid: Ministerio de Fomento (2018).

Disponible en: http://www.puertos.es/es-es/estadisticas/Paginas/MemoriasAnuales.aspx

(Consultada en mayo de 2018)

METCALFE, S. (1995). "The Economic Foundations of Technology Policy: Equilibrium and Evolutionary Perspectives": En Paul Stoneman (Ed) Handbook of the economics of innovation and technological change (pp. 409-512). Warwick: Wiley-Blackwell.

NAIDER (2012). "Estudio sobre los efectos de la I+D en los resultados empresariales para España". Fundación Española para la Ciencia y la Tecnología (FECYT). Disponible en: https://icono.fecyt.es/sites/default/files/filepublicaciones/informe_efectos_innovacion_0.pdf [Consultada el 10 de Mayo de 2018]. 
OECD/Eurostat (2018): Oslo Manual 2018: Guidelines for Collecting, Reporting and Using Data on Innovation, 4th Edition, The Measurement of Scientific, Technological and Innovation Activities, OECD Publishing, Paris/Eurostat, Luxembourg, https://doi.org/10.1787/9789264304604-en.

OECD (2015): Frascati Manual 2015: Guidelines for Collecting and Reporting Data on Research and Experimental Development. Paris: OECD Publishing.

O'MAHONY, M. y VECCHI, M. (2009): "R\&D, knowledge spillovers and company productivity performance". Research Policy, 38(1): pp. 35-44.

PUERTOS DEL ESTADO (2018). Puertos del Estado. Madrid: Ministerio de Fomento (2018). Disponible en: http://www.puertos.es/es-es/nosotrospuertos/Paginas/Nosotros.aspx

[Consultada el 10 de Mayo de 2018].

PLAN GENERAL DE CONTABILIDAD (2007). Real Decreto 1514/2007 de 16 de noviembre. Boletín Oficial del Estado, núm 278, de 20 de noviembre de 2007.

Disponible en: https://www.boe.es/boe/dias/2007/11/20/pdfs/C00001-00152.pdf

RIVERO TORRE, P. (1996). Análisis de balances y estados complementarios, Madrid: Pirámide.

ROMER, P. M. (1986). "Increasing returns and long-run growth". Journal of Political Economy, 94(5), pp. 10021037.

SÁNCHEZ ARROYO, G. (2002). Ejercicios de contabilidad y análisis e interpretación de la información contable. Madrid: Pirámide.

SÁNCHEZ, M.P. y SALAZAR, J. C. (2010). El papel de la innovación en el nuevo modelo económico español: Editado por Accenture SL en el marco de la Cátedra UAM-Accenture de Economía y Gestión de la Innovación.

SOMOHANO RODRÍGUEZ, FM.; LÓPEZ FERNÁNDEZ, J.M. y MARTÍNEZ GARCÍA, F.J. (2018). "The effect of innovation on business performance in economic downturn. An application to the automotive industry" Revista de Contabilidad, (21)1, pp. 91-105. https://doi.org/10.1016/j.rcsar.2017.11.001

SOLOW, R.M. (1957). "Technical change and the aggregate production function". The Review of Economics and Statistics, 39(3), pp. 312-320. 